\title{
Clinical Study \\ Clinical Finding and Thyroid Function in Women with Struma Ovarii
}

\author{
Ali Hosseini ${ }^{1}$ and Aida Moeini ${ }^{1,2}$ \\ ${ }^{1}$ Department of Obstetrics and Gynecology, Shahid Beheshti University of Medical Science, Tehran, Iran \\ ${ }^{2}$ Department of Obstetrics and Gynecology, Tajrish Hospital, Tajrish Square, Tehran 1989934148, Iran
}

Correspondence should be addressed to Aida Moeini; a.moeini64@gmail.com

Received 29 March 2013; Accepted 8 May 2013

Academic Editor: Mario Budroni

Copyright (C) 2013 A. Hosseini and A. Moeini. This is an open access article distributed under the Creative Commons Attribution License, which permits unrestricted use, distribution, and reproduction in any medium, provided the original work is properly cited.

Background. Struma ovarii (SO) is a variant of dermoid tumors which completely or mainly composed of thyroid tissues. Objective. We report our experience in the diagnosis and thyroid function of patients with SO in our hospital and also review the management and treatment option of this tumor. Materials and Methods. Between 2000 and 2012, 15 consecutive females with SO who were presented to our hospital were fully assessed. All women had histologically confirmed struma ovarii. The medical records of all patients including presenting symptoms, CT scan finding, and hormonal levels were collected for final analysis. Results. Average patient age was 36.6 years (ranging from 21 to 69 ). The mean \pm SDs of serum TSH, T4, and T3 were $2.16 \pm 2.4 \mathrm{mUI} / \mathrm{mL}, 6.21 \pm 3.74 \mathrm{ng} / \mathrm{dL}$, and $82.1 \pm 40.5 \mathrm{ng} / \mathrm{dL}$, respectively. The value of TSH was lower than normal value in $26.7 \%$. Also, antithyroglobulin and anti-TPO were positive in 2 and one cases, respectively. Conclusion. Based on our data, it is more likely to see a disturbance in serum values of thyroid function test in women with SO.

\section{Introduction}

Struma ovarii (SO) is a variant of dermoid tumors of the ovary in which thyroid tissue components is the major constituent [1]. It is of germ cell origin and accounts for about $2 \%$ of all ovarian germ cell tumors [2]. This neoplasm is predominantly found in women between the ages of 40 and 60 years. They are composed of mature thyroid tissue-which mostly (approximately $95 \%$ ) has a benign nature-occupying more than 50\% of the mass [3-5]. Thyroid tissue in the teratoma can exhibit all histological and pathological patterns of normal thyroid epithelium. In major proportion of patients, this neoplasm is nonfunctional and only 5\% to $15 \%$ reveal hyperthyroidism. Immunohistochemical staining for thyroglobulin may be required to identify the cells' origin. Most of these tumors are asymptomatic, and diagnosed after surgery of a pelvic mass $[6,7]$. It is also difficult to distinguish between struma ovarii and dermoid cysts on the basis of their sonographic appearance [5]. Because of the rarity of the tumor, only a few reports with detailed data on thyroid function of the patient have been published. We report our experience in the diagnosis and thyroid function of patients with SO in our hospital and also review the management and treatment option of this tumor.

\section{Materials and Methods}

Between 2000 and 2012, 15 consecutive females with SOs who were presented to our hospital were fully assessed. All women had histologically confirmed struma ovarii. All patients provided an informed written consent; the study was approved by Ethics Committee of Tajrish Hospital.

All patients were subjected to the standard gynecologic evaluation, including history taking and clinical examination. Baseline serum samples were taken to assess thyroid hormone (T3, T4), thyroid stimulating hormone (TSH), antithyroglobulin, and anti-TPO, as well as serum levels of alpha fetoprotein (AFP), beta human chorionic gonadotropin (B-HCG), CA-125, and CEA laboratory tests. Also, all cases underwent CT examination with speed 16-slice spiral CT at slice thickness at $5 \mathrm{~mm}$, layer interval at $5 \mathrm{~mm}$. 


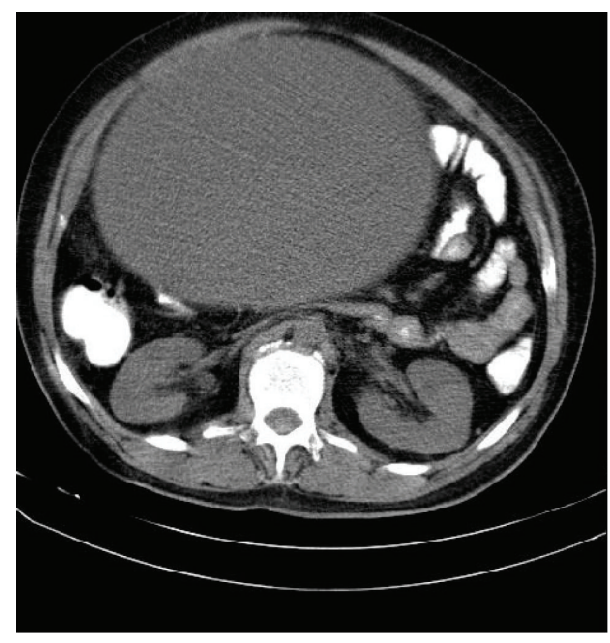

(a)

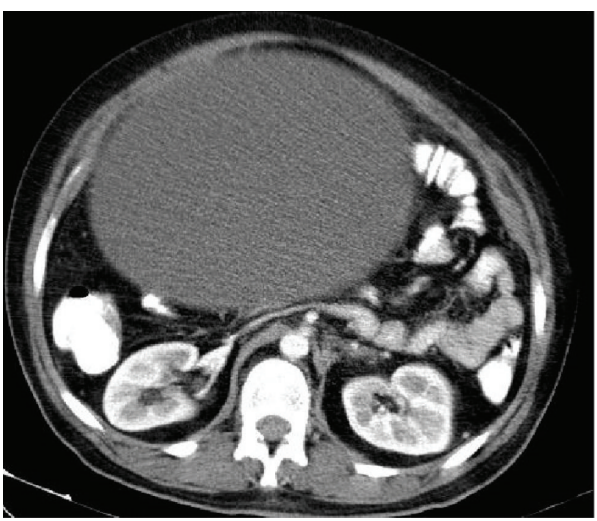

(c)

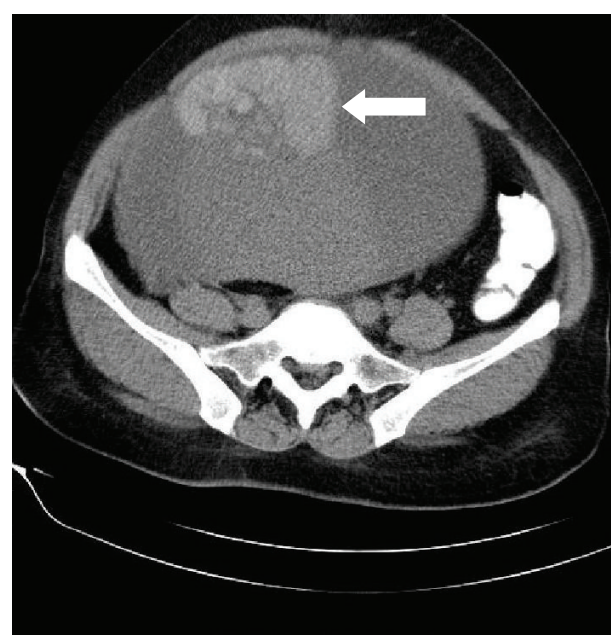

(b)

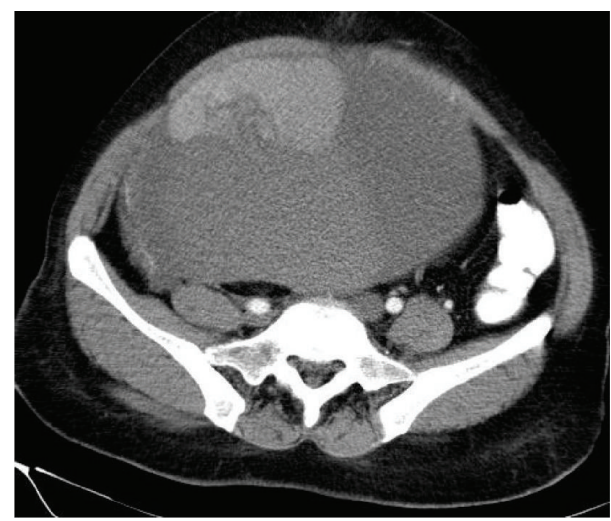

(d)

FIGURE 1: Axial CT image of large cystic abdominal mass; ( $a$ and b) Noncontrast-enhanced CT image shows a mass with soft tissue components and multiple septations (white arrow); (c and d) After intravenous bolus of contrast medium soft tissue components showed enhancement within the mass.

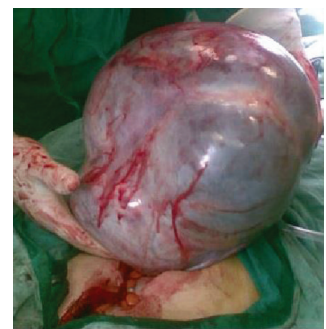

(a)

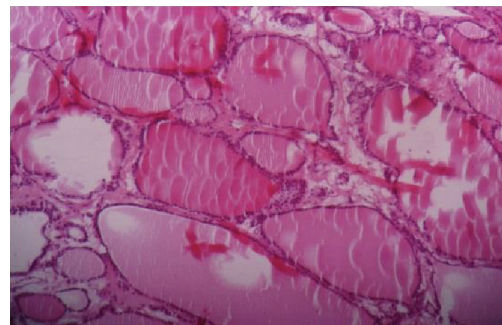

(b)

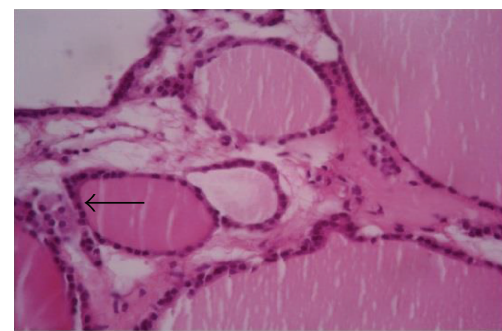

(c)

FIGURE 2: (a): The surgically excised ovarian tumor; (b) Low-magnification view of benign thyroid tissue follicles of various sizes in the ovary stained with hematoxylin and eosin; (c) High-magnification view of a benign follicles lined by flat-to-cuboidal cells (black arrow).

The medical records of all patients were collected for final analysis.

\section{Results}

Between 2000 and 2012, fifteen patients were included in this study. Average patient age was 36.6 years (ranging from 21 to 69) and all were followed up for a mean of 4 years (ranging from 1 to 13 years). Presenting symptoms of SO at the time of diagnosis were lower abdominal pain in $3(20 \%)$, palpable lower abdominal mass in $3(20 \%)$, abnormal vaginal bleeding in $2(13.3 \%)$, and no definite presenting symptoms in the remaining 7 patients (46.7\%), and the presence of an ovarian tumor was an incidental finding by imaging. Duration of symptoms ranged from one to 12 months. 
According to CT scan, 13 were unilateral (86.7\%): 7 in the left ovary and 6 in the right side and 2 cases had bilateral lesion $(13.3 \%)$. The diameters of tumors ranged from 2.5 to $25.0 \mathrm{~cm}$ (Figure 1), with an average of $7.6 \mathrm{~cm}$. Finding of noncontrast CT images reveals foci of high attenuation in 12 tumors (80\%); also curvilinear calcifications were recognized in the thickened septi or cyst walls of 8 women (53.4\%). No evidence of metastasis or peritoneal implants was noted in patients.

Laboratory data on admission of women revealed no abnormality value in blood cell count, serum creatinine level, and erythrocyte sedimentation rate (ESR). Tumor marker CA- 125 was raised (reference value $<35 \mathrm{U} / \mathrm{mL}$ ) in 4 cases (26.7\%), but the results of AFP, B-HCG, and CEA laboratory tests were all in normal range. The mean \pm SDs of serum TSH, $\mathrm{T} 4$, and T3 were $2.16 \pm 2.4 \mathrm{mUI} / \mathrm{mL}, 6.21 \pm 3.74 \mathrm{ng} / \mathrm{dL}$, and $82.1 \pm 40.5 \mathrm{ng} / \mathrm{dL}$, respectively. The value of TSH was lower than normal value (normal: $0.5-4.2 \mathrm{mUI} / \mathrm{mL}$ ) in 4 patients (26.7\%). Also, antithyroglobulin and anti-TPO were positive in 2 and one cases, respectively.

3.1. Pathologic Findings. In macroscopic study, the tumors were pale in color, with smooth surface, showing nodular protrusions and contained with clear serous fluid and gelatinous material. In microscopic view, lesions had normal looking thyroid tissue composed of variable size follicles filled with colloid material (Figure 2). With the use of immunohistochemistry, the cyst lining was shown to express thyroglobulin. Malignant struma ovarii was present in 2 of the 15 (13.3\%) patients. Both cases had positive CA-125 and anti-TPO.

\section{Discussion}

Boettlin who was first to describe SO in 1889 as the presence of thyroid follicular tissue in ovaries, and after that Gottschalk published further reports [6]. In fact, SO consists of tumor which is completely or mainly composed of thyroid tissues, as well as some teratomas with less than $50 \%$ thyroid tissues, but thyroid tissue could be easily identified [7]. This thyroid tissue can reveal all histological and pathological patterns of normal thyroid epithelium, such as an adenoma or as papillary or follicular carcinoma. It may also organize in solid, embryonal, or pseudotubular patterns [3]. Malignant transformation is infrequent, only about $5 \%$ of SO being malignant. Metastases are found in 5\%-6\% of patients with malignant SO [5].

SO may be diagnosed at any age, even in children, but it occurred mostly in reproductive years of patients [4]. This tumor generally presents with nonspecific symptoms that are analogous to those of other ovarian neoplasms. Diagnosis is difficult unless the tumor is very large or causes remarkable thyrotoxicosis. In $5 \%$ to $12 \%$ of cases, functional thyroid tissue may be present in SO; and classic symptoms of hyperthyroidism may be evident [8]. The functional thyroid tissue in the ovary may represent the patient's only functional thyroid tissue, and hypothyroidism may occur after resection [9]. Yoo and colleagues reviewed medical records of 25 patients with histologically confirmed SO and showed that the most common presenting symptoms at the time of diagnosis were lower abdominal pain, followed by palpable lower abdominal mass, and abnormal vaginal bleeding. However, the presence of an ovarian tumor was found incidentally by ultrasonography in nearly half of patients [6]. The widely accepted tumor marker of ovarian cancers, CA-125, is found to be increased in this neoplasm; however, Yoo et al. stated that its measurement was of little clinical value in these patients $[6,10]$. The diagnostic criterion of $\mathrm{SO}$ is based on histological features of the resected ovary. However, immunohistochemical staining for thyroglobulin may be required to identify the cells' origin [6].

SO tumors vary in size but usually measure less than $10 \mathrm{~cm}$ in diameter, are often unilateral, and may be associated with a mature cystic teratoma in the same or contralateral ovary as seen in our cases [5]. In the two reports by Ezon et al. and Savelli et al., the largest dimensions were 30 and $20 \mathrm{~cm}$, respectively $[4,5]$. One of our patients also had a tumor size of $25 \mathrm{~cm}$ in largest dimension.

Savelli et al. describe that the most specific sonographic feature of pure SO was the "struma pearl" in their series of 31 women. Magnetic resonance imaging (MRI) and CT findings in SO were explained by Ikeuchi and colleagues. MR findings include a multicystic mass with a multilobulated surface, some solid components including thickened septi or cyst walls, and cystic components showing a variety of signal intensities on both T1- and T2-weighted images. Also, CT findings are characterized by the presence of high attenuation area and calcifications [5].

Because of the paucity of $\mathrm{SO}$, there is no clarity regarding the proper management of these patients [4]. Historically, the decision has been largely influenced by the size of the tumor and concern for possible malignancy. Patients with benign $\mathrm{SO}$ are cured by surgical removal of the tumor [11]. If ascites and/or pleural effusion are present, they usually disappear after surgery. In fact, metastasis is not characteristic for benign SO, but recurrence is a rare condition [5]. After treatment, the prognosis of benign strumosis and of malignant SO without metastases is good. Many authors provide followup from 4 months to 3 years for patients with benign, nonfunctional disease [4]. Careful postoperative monitoring for any signs of hypothyroidism is mandated because the thyroid tissue present in the ovary may be the patient's only functional thyroid tissue [9].

\section{References}

[1] R. D. Kempers, M. B. Dockerty, D. L. Hoffman, and L. G. Bartholomew, "Struma ovarii: ascitic, hyperthyroid, and asymptomatic syndromes," Annals of Internal Medicine, vol. 72, no. 6, pp. 883-893, 1970.

[2] P. Rotman-Pikielny, J. C. Reynolds, W. C. Barker, P. M. Yen, M. C. Skarulis, and N. J. Sarlis, "Recombinant human thyrotropin for the diagnosis and treatment of a highly functional metastatic struma ovarii," Journal of Clinical Endocrinology and Metabolism, vol. 85, no. 1, pp. 237-244, 2000.

[3] N. C. Bozkurt, B. Karbek, E. C. Ozkaya, E. Cakal, and T. Delibaşi, "Struma ovarii presenting with Hashimoto's thyroiditis: a case report," Journal of Medical Case Reports, vol. 12, article $572,2011$. 
[4] I. Ezon, N. Zilbert, L. Pinkney, J. J. Wei, R. Malik, and E. P. Nadler, "A large struma ovarii tumor removed via laparoscopy in a 16-year-old adolescent," Journal of Pediatric Surgery, vol. 42, no. 8, pp. e19-e22, 2007.

[5] L. Savelli, A. C. Testa, D. Timmerman, D. Paladini, O. Ljungberg, and L. Valentin, "Imaging of gynecological disease (4): clinical and ultrasound characteristics of struma ovarii," Ultrasound in Obstetrics and Gynecology, vol. 32, no. 2, pp. 210-219, 2008.

[6] S. C. Yoo, K. H. Chang, M. O. Lyu, S. J. Chang, H. S. Ryu, and H. S. Kim, "Clinical characteristics of struma ovarii," Journal Of Gynecologic Oncology, vol. 19, no. 2, pp. 135-138, 2008

[7] J. Shen, X. Xia, Y. Lin, W. Zhu, and J. Yuan, "Diagnosis of Struma ovarii with medical imaging," Abdominal Imaging, vol. 36, no. 5, pp. 627-631, 2011.

[8] T. Dunzendorfer, A. de Las Morenas, T. Kalir, and R. M. Levin, "Struma ovarii and hyperthyroidism," Thyroid, vol. 9, no. 5, pp. 499-502, 1999.

[9] J. P. Carvalho, F. M. Carvalho, F. F. Lima de Oliveira, and R. Y. Asato de Camargo, "Hypothyroidism following struma ovarii tumor resection: a case report," Revista do Hospital das Clinicas de Faculdade de Medicina da Universidade de Sao Paulo, vol. 57, no. 3, pp. 112-114, 2002.

[10] W. D. Salman, M. Singh, and Z. Twaij, "A case of papillary thyroid carcinoma in struma ovarii and review of the literature," Pathology Research International, vol. 2010, Article ID 352476, 5 pages, 2010.

[11] S. F. Gould, R. L. Lopez, and W. C. Speers, "Malignant struma ovarii. A case report and literature review," Journal of Reproductive Medicine for the Obstetrician and Gynecologist, vol. 28, no. 6, pp. 415-419, 1983. 


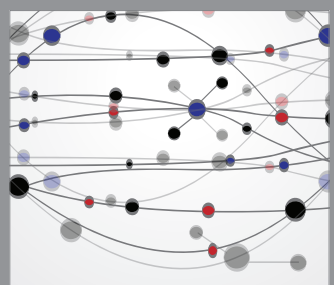

The Scientific World Journal
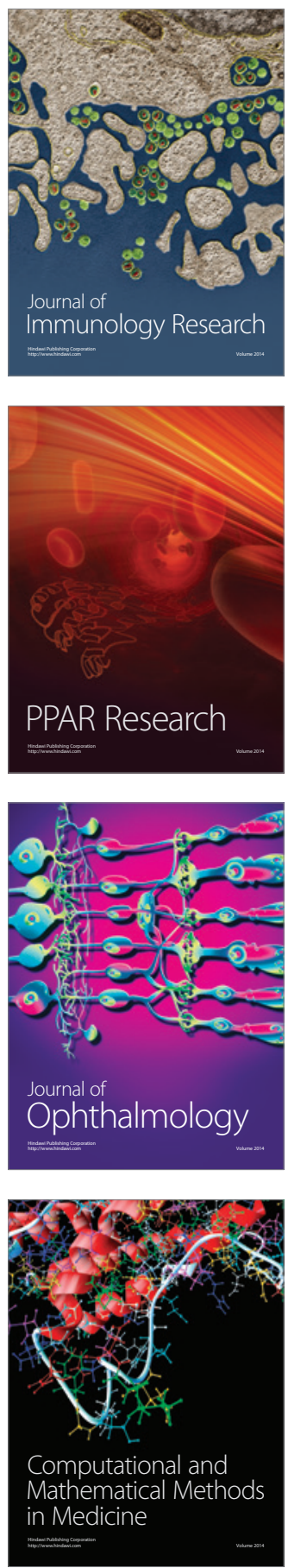

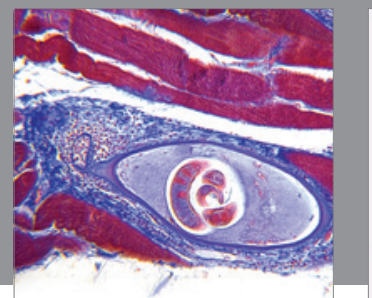

Gastroenterology

Research and Practice
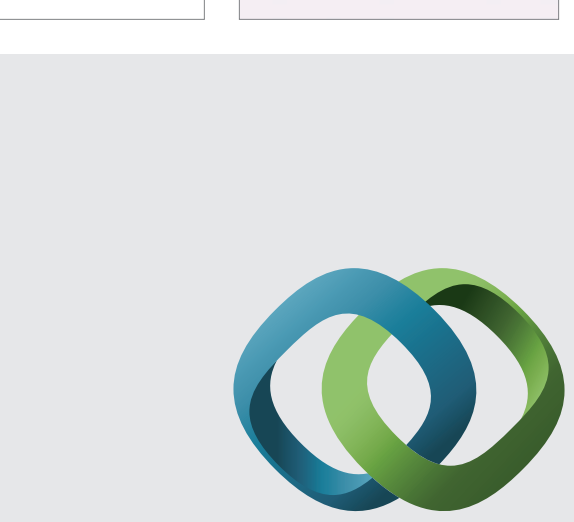

\section{Hindawi}

Submit your manuscripts at

http://www.hindawi.com
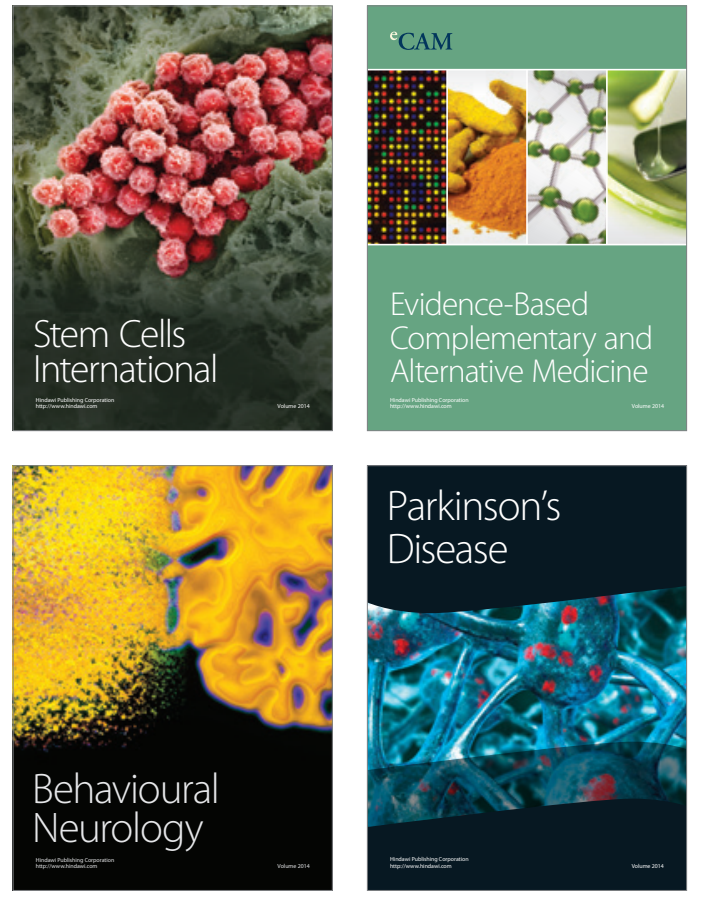
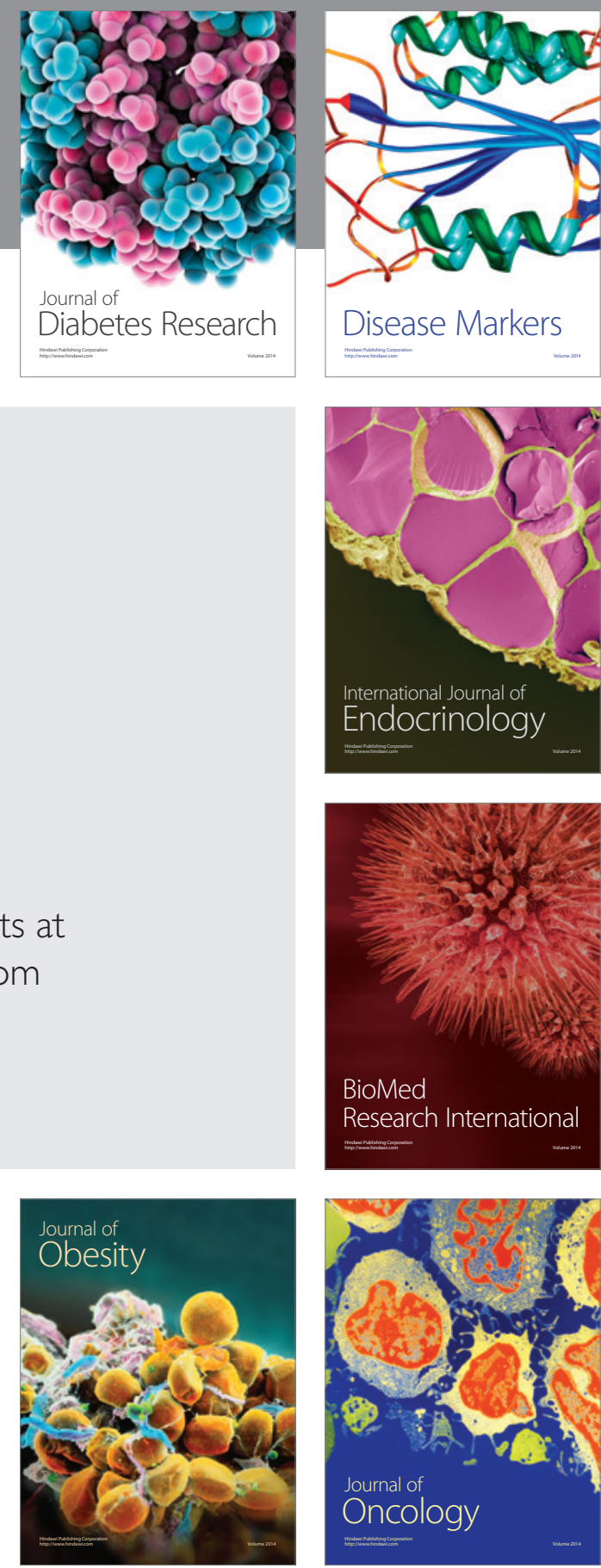

Disease Markers
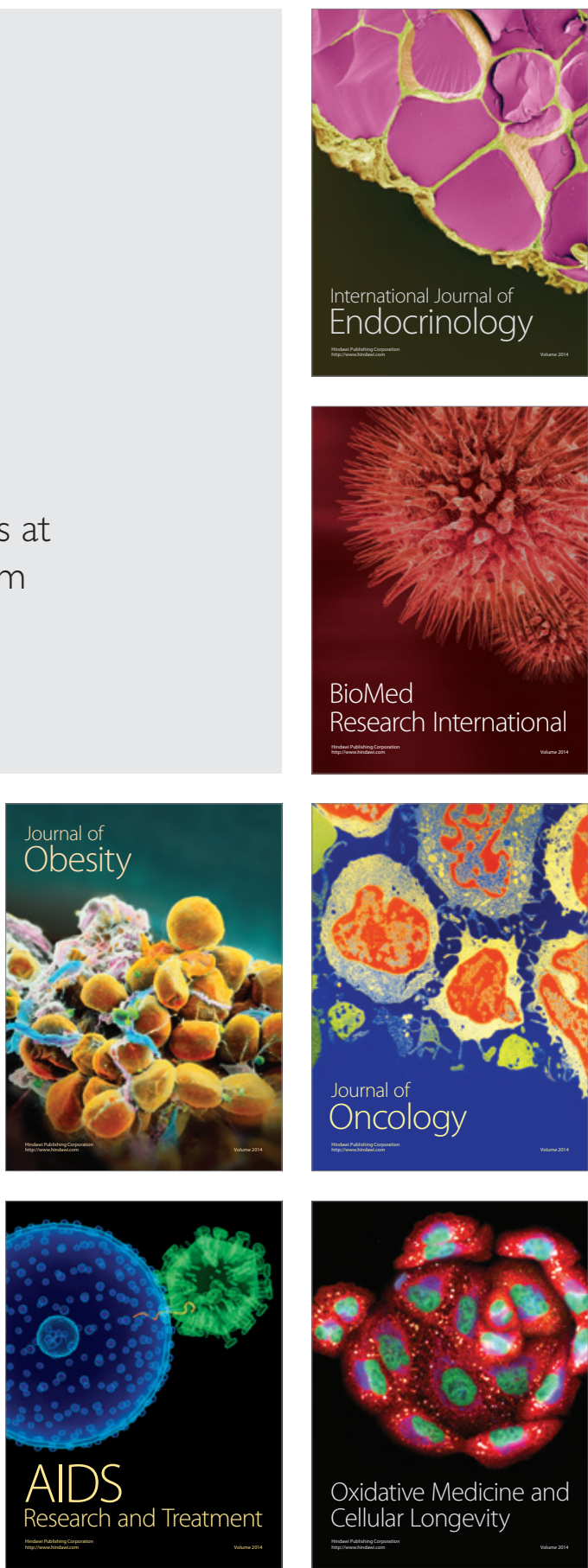